\title{
Systematischer Index
}

Klasse Reptilia
Ordnung Testudines
Unterordnung Cryptodira

Fam. Chelydridae

\section{Chelydra}

Ch. serpentina

Ch. s. serpentina

Ch. s. acutirostris

Ch. s. osceola

Ch. $s$ rossignonii

\section{Macroclemys}

M. temminckii

Fam. Kinosternidae

\section{Claudius}

C. angustatus

\section{Kinosternon \\ $K$. abaxillare \\ K. acutum \\ $K$. angustipons \\ K. baurii}

\section{K. b. baurii}

K. b. palmarum

K. creaseri

K. cruentatum

K. dunni

$K$. flavescens
$K$. f. flavescens
K. f. spooneri
K. f. stejnegeri

$K$. herrerai

$K$. hirtipes
$K$. h. hirtipes
K. h. murrayi

K. leucostomum 7

K. mopanum $\quad 8$

K. postinguinale $\quad 8$

K. scorpioides 8

K. s. scorpioides 8

$K$. s albogulare $\quad 9$

K. s. integrum 9

K. s. pachyrum $\quad 9$

K. s. panamense $\quad 9$

K. s seriei 10

$K$. sonoriense $\quad 10$

K. spurrelli $\quad 10$

K. subrubrum 10

K. s. hippocrepis 11

K. s. steindachneri $\quad 11$

Staurotypus 11

S. salvini $\quad 11$

$\begin{array}{ll}S . & \text { triporcatus } \\ & 12\end{array}$

Sternotherus 12

S. carinatus 12

S. minor 13

S. m. minor 13

S. m. depressus 13

S. m. peltifer 13

S. odoratus 13

Fam. Dermatemydidae $\quad 14$

Dermatemys 14

D. mawii 14

Fam. Platysternidae $\quad 15$

Platysternon 15

P. megacephalum 15

P. m. megacephalum $\quad 15$

P. m. peguense $\quad 15$

$\begin{array}{ll}\text { P. m. vogeli } & 16\end{array}$ 
Fam. Emydidae

Batagur

B. baska

Callagur

C. borneoensis

Chinemys

Ch. kwangtungensis

Ch. megalocephala

Ch. reevesii

Chrysemys

Ch picta

Ch. p. picta

Ch. p. bellii

Ch. p. dorsalis

Ch. p. marginata

\section{Clemmys}

C. guttata

C. insculpta

C. marmorata

C. m. marmorata

C. m. pallida

C. muhlenbergii

\section{Cuora}

C. amboinensis

C. flavomarginata

C. galbinifrons

C. trifasciata

C. vunnanensis

Cyclemys

C. dentata

Deirochelys

D. reticularia
D. r. reticularia
D. r. chrysea
D. r. miaria

Emydoidea

E. blandingii

Emys

E. orbicularis
Geoclemys

G. hamiltonii

Geoemyda

G. annulata 29

G. areolata 29

G. depressa 29

G. funerea 30

G. grandis 30

G. leytensis 30

G. pulcherrima 30

G. p. pulcherrima 30

G. p. incisa 31

G. p. manni

G. punctularia 31

G. p. punctularia

G. p. diademata

G. p. lunata 32

G. p. melanosterna 32

G. p. nasuta 32

G. nubida 33

G. r. rubida 33

G. r. perixantha 33

G. silvatica 33

G. spengleri 33

G. s. spengleri 33

G. s japonica 34

G. spinosa 34

G. tricarinata 34

G. trijuga 35

G. t. trijuga 35

G. t. coronata 35

G. t. edeniana 35

G. t. indopeninsularis 35

G. t. parkeri 36

G. t. thermalis 36

Graptemys 36

G. barbouri 36

G. caglei 37

G. flavimaculata 37

G. geographica $\quad 37$

G. kohnii 37

G. nigrinoda 38

G. n. nigrinoda 38

G. n. delticola 38

G. oculifera $\overline{38}$

G. pseudogeographica $\quad 38$ 
G. p. pseudogeographica

G. p. ouachitensis

G. p. sabinensis

G. p. versa

G. pulchra

\section{Hardella}

H. thurii

H. t. turiii

H. t. indi

Hieremys

H. annandalii

Kachuga

$K$. dhongoka

K. kachuga

$K$. smithii

$K$. sylhetensis

K. tecta
K. $t$. tecta
$K$. t. circumdata
$\kappa$. $t$. tentoria

K. trivittata

\section{Malaclemys \\ M. terrapin \\ M. t. terrapin \\ M. $t$. centrata \\ M. $t$. littoralis \\ M. t. macrospilota \\ M. t. pileata \\ M. t. tequesta \\ M. t. rhizophorarum}

\section{Malayemys \\ M. subtrïuga}

\section{Mauremys}

M. bealei

M. caspica

M. c. caspica

M. c. leprosa

$M$. c. rivulata

$M$. japonica

$M$. nigricans

\section{Morenia}

M. ocellata

$M$. petersi
38

39

39

39

39

40

40

40

40

40

40

41

41

42

42

42

42

42

43

43

43

44

44

44

45

45

45

45

46

46

46

46

47

47

47

47

48

48

49

49

50

50

50
Notochelys

$N$. platynota

Ocadia

O. sinensis

Orlitia

O. borneensis

51

51

51

51

51

52

Pseudemys 52

P. concinna 53

P. c. concinna 53

P. c. hieroglyphica 53

P. c. hiltoni 53

P. c. mobilensis 53

P. c. suwanniensis $\quad 54$

P. c. texana 54

P. dorbigni $\quad 54$

P. d. dorbigni $\quad 54$

P. d. brasiliensis $\quad 55$

P. floridana 55

P. f. floridana 55

P. f. hoyi 55

P. f. peninsularis $\quad 55$

P. grayi $\quad 55$

P. ornata 56

P. o. ornata $\quad 56$

P. o. callirostris $\quad 56$

P. o. nebulosa 57

P. o. yaquia 57

P. rubriventris $\quad 57$

$P$. r. rubriventris $\quad 57$

P. r. alabamensis $\quad 57$

P. r. bangsi 58

P. r. nelsoni 58

$\begin{array}{ll}\text { P. scripta } & 58\end{array}$

P. s. scripta 58

P. s. elegans $\quad 58$

P. s. gaigeae 59

P. s. taylori 59

P. s. troostii 59

P. terrapen $\quad 59$

P. $t$. terrapen $\quad 59$

P. $t$. decorata 60

P. $t$. granti 60

P. t. malonei $\quad 60$

P. $t$. rugosa 60

P. $t$. stejnegeri 61

P. t. vicina 61 
XXIV

Pyxidea

P. mouhotii

Siebenrockiella

$S$. crassicollis

\section{Terrapene}

T. carolina

T. c. carolina

T. c. bauri

T. c. major

T. c. mexicana

T. c. triunguis

T. c. yucatana

T. coahuila

T. nelsoni

T. $n$. nelsoni

T. $n$. klauberi

T. ornata

T. o. ornata

T. o. luteola

Fam. Test udinidae

Gopherus

G. agassizii

G. berlandieri

G. flavomarginatus

G. polyphemus

\section{Homopus}

H. areolatus

$H$. boulengeri

$H$. femoralis

H. signatus

Kinixys

$K$. belliana
$K$. b. belliana
K. b. mertensi
$K$. b. nogueyi

$K$. erosa

$K$. homeana

Malacochersus

M. tornieri

Pyxis

$P$. arachnoides
61 Testudo

T. angulata

T. carbonaria

62

62

T. chilensis

T. denticulata

T. elegans

62

63

63

64

64

64

65

65

65

66

66

66

66

66

66

T. elephantopus

T. e. elephantopus

T. e. abingdonii

T. e. chathamensis

T. e. danwini

T. e. ephippium

T. e. galapagoensis

$T$. e. hoodensis

T. e. nigrita

T. e phantastica

T. e. wallacei

T. elongata

T. emys

T. forstenii

$T$. geometrica

T. gigantea

T. g. gigantea

67

67

67

67

68

68

68

68

69

69

69

T. g. daudinii

T. g. elephantina

T. graeca

T. g. graeca

T. g. ibera

T. g. terrestris

T. g. zarudnyi

T. hermanni

T. $h$ hermanni

T. $h$ robertmertensi

T. horsfieldii

T. impressa

T. kleinmanni

$70 \quad T$. marginata

$70 \quad T$. oculifera

$70 \quad T$. pardalis

T. p. pardalis

T. p. babcocki

$T$. planicauda

T. platynota

$T$. radiata

72

72

72

T. sulcata

T. tentoria

T. t. tentoria

T. $t$. trimeni

T. t. verroxii 
T. travancorica

T. yniphora

Fam. Cheloniidae

Caretta

C. caretta

Chelonia

Ch. depressa

Ch. mydas

Ch. m. mydas

Ch. m. japonica

Eretmochelys

E. imbricata

E. i. imbricata

E. i. bissa

Lepidochelys

L. kempii

L. olivacea

Fam. Dermochelydidae 99

Dermochelys

D. coriacea

Fam. Carettochelydidae 100

Carettochelys

C. insculpta

Fam. Trionychidae

Chitra

Ch. indica

Cyclanorbis

C. elegans

C. senegalensis

Cycloderma

C. aubryi

C. frenatum

Lissemys

L. punctata

L. p. punctata

L. p. granosa

L. p. scutata
99

99

93

93

93

93

94

94

95

95

95

96

96

97

97

97

97

98

98

9

100

100

100

100

100

101

101

101

102

102

102

102

103

103

103

103
Pelochelys

P. bibroni

Trionyx

T. ater

T. cartilagineus

T. euphraticus

T. ferox

T. formosus

T. gangeticus

T. hurum

T. leithii

T. muticus

T. m. muticus

T. m. calvatus

T. nigricans

T. sinensis

T. spiniferus

T. s. spiniferus

T. s. asper

T. s. emoryi

T. s. guadalupensis

T. s. hartwegi

T. s. pallidus

T. steindachneri

T. subplanus

T. triunguis

Unterordnung Pleurodira

Fam. Pelomedusidae

Pelomedusa

P. subrufa

Pelusios

P. a. nanus

P. carinatus

P. castaneus

P. gabonensis

$P$. niger

$P$. sinuatus

P. subniger

P. williamsi

P. w. williamsi

P. w. hutescens
114

114

114

115

115

116

116

116

104

104

104

106

107

108

108

109

109

109

109

110

110

111

111

112

113

113

113

113

113

114

116

116

117

117

118

118

119

119

119 
Podocnemis

P. dumeriliana

P. erythrocephala

$P$. expansa

$P$. lewyana

P. madagascariensis

P. sextuberculata

$P$. unifilis

P. vogli

Fam. Chelidae

Chelodina

Ch. expansa

Ch. longicollis

Ch. l. longicollis

Ch. l. sulcifera

Ch. novaeguineae

Ch. oblonga

Ch. rugosa

Ch. siebenrocki

Ch. steindachneri

Chelus

Ch. fimbriatus

Elseya
E. dentata
E. latisternum
E. novaeguineae

Emydura

E. albertisii

E. australis

E. krefftii

E. macquarrii

E. m. macquarrii

E. m. signata

E. subglobosa

\section{Hydromedusa \\ H. maximiliani \\ H. tectifera}

\section{Phrynops}

P. geoffroanus

P. g. geoffroanus

P. g. tuberosus

P. gibbus
119

119

120

120

121

121

121

121

122

122

122

122

122

122

123

123

123

123

124

124

124

125

125

125

126

126

126

127

127

127

128

128

128

128

129

129

129

129

130

130

130

131
P. hilarii

131

131

P. hogei

132

$P$. nasutus

132

P. n. nasutus

132

P. n. wermuthi 132

P. nufipes

132

P. tuberculatus

133

P. $t$. tuberculatus

P. $t$. vanderhaegei

133

133

Platemys

133

P. pallidipectoris

133

$P$. platycephala

$P$. radiolata

P. spixii

133

134

134

Pseudemydura

134

P. umbrina

135

Ordnung Crocodylia $\quad 135$

Fam. Alligatoridae $\quad 135$

Alligator

135

A. mississippiensis

135

A. sinensis

136

Caiman

136

C. crocodilus $\quad 136$

C. c. crocodilus 136

C. c. apaporiensis $\quad 137$

C. c. fuscus 137

C. c. matogrossiensis $\quad 137$

C. c. paraguayensis $\quad 138$

C. c. yacare 138

C. latirostris $\quad 138$

C. l. latirostris $\quad 138$

C. l. chacoensis 139

Melanosuchus

139

$M$. niger

139

Paleosuchus

139

P. palpebrosus $\quad 140$

P. trigonatus

140 
XXVII

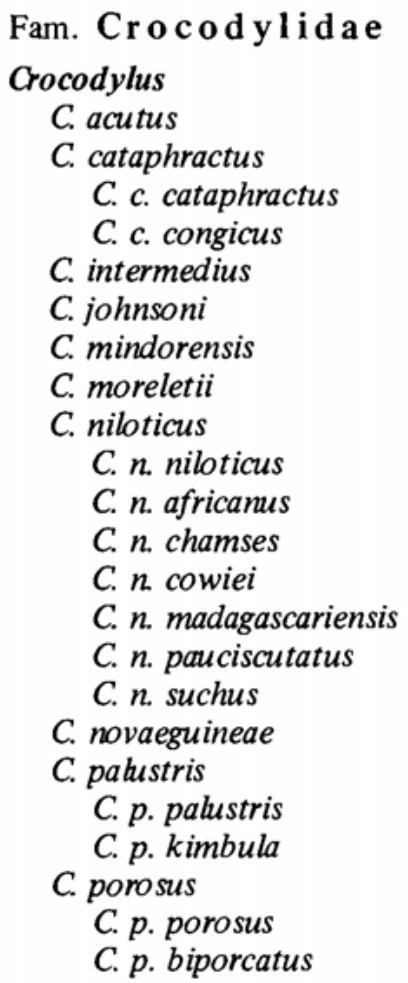

140

140

141

142

142

142

142

143

143

143

143

143

144

144

144

145

145

145

146

146

146

146

147

147

147
C. rhombifer

C. siamensis

Osteolaemus

O. tetraspis

O. t. tetraspis

o. t. osborni

Tomistoma

T. schlegelii

Fam. Gavialidae

Gavialis

G. gangeticus

Ordnung Rhynchocephalia

Fam. Sphenodontidae

Sphenodon

S. punctatus

S. p. punctatus

S. p. guntheri

S. p. reischeki
150

150

150

150

150

150

151

151

148

148

148

148

148

149

149

149

151

151 
